Supporting Information

\title{
Solution and Solid-State Characterization of PbSe
}

\section{Precursors}

Prathamesh B. Vartak, ${ }^{[a]}$ Zhongyong Wang, ${ }^{[a]}$ Thomas L. Groy, ${ }^{[b]}$ Ryan J. Trovitch, ${ }^{[b]}$ and Robert Y. Wang*[a]

[a] School of Engineering for Matter, Transport and Energy, Arizona State University, Tempe, Arizona 85287-6106, United States.

[b] School of Molecular Sciences, Arizona State University, Tempe, Arizona 85287-1604, United States. 


\section{Contents:}

Figure S1 Integration of the ${ }^{1} \mathrm{H}$ NMR resonances of $\mathbf{1}$ in DMSO- $d_{6}$. S3

Figure $\mathrm{S} 2{ }^{13} \mathrm{C}$ NMR spectrum of 1 in DMSO- $d_{6}$.

Figure S3 Stacked ${ }^{13} \mathrm{C}$ NMR spectra of 1 , diphenyl diselenide, and ethylenediamine in DMSO- $d_{6}$. S4

Figure $S 4{ }^{77}$ Se NMR spectrum of 1 and diphenyl diselenide in DMSO- $d_{6}$.

Figure S5 Comparison of the experimental and simulated isotopic pattern of the $\left[\mathrm{PbSe}_{3} \mathrm{C}_{18} \mathrm{H}_{15}\right]^{-1}$ ion. $\mathrm{S} 5$

Figure $\mathrm{S} 6$ Mass spectra of the PbSe precursor with and without the addition of potassium iodide. S6

Figure S7 Packing in the crystal structure of $2 . \quad S 7$

Figure $\mathrm{S} 8{ }^{1} \mathrm{H}$ NMR spectrum of aged $\mathbf{2}$ dissolved in DMSO- $\boldsymbol{d}_{6}$. S8

Figure $\mathrm{S} 9{ }^{13} \mathrm{C}$ NMR spectrum of aged 2 dissolved in DMSO- $d_{6}$. S9

Figure S10 Schematic of 1-D chain in the central region of the unit cell of crystal structure of $3 . \quad$ S10

Figure S11 Optical micrograph and SEM images of 3. S10

Figure $\mathrm{S} 12{ }^{1} \mathrm{H}$ NMR spectrum of $\mathbf{3}$ dissolved in DMSO- $d_{6} . \quad$ S11

Figure $\mathrm{S} 13{ }^{13} \mathrm{C}$ NMR spectrum of $\mathbf{3}$ dissolved in DMSO- $d_{6}$. $\quad$ S11

Discussion S1: Crystal structure of 3 showing 1-D molecular chains. S9

Table S1 Additional details of data collection and refinement for 2 . S12

Table S2 Atomic coordinates and equivalent isotropic atomic displacement parameters $\left(\AA^{2}\right)$ for $\mathbf{2} . \quad$ S12

Table S3 Bond lengths $(\AA)$ for $2 . \quad S 14$

Table S4 Bond angles $\left({ }^{\circ}\right)$ for $2 . \quad S 15$

Table S5 Torsion angles $\left({ }^{\circ}\right)$ for $\mathbf{2} . \quad S 17$

Table S6 Anisotropic atomic displacement parameters $\left(\AA^{2}\right)$ for $\mathbf{2}$ S18

Table S7 Hydrogen atomic coordinates and isotropic atomic displacement parameters $\left(\AA^{2}\right)$ for $\mathbf{2}$. S20

Table S8 Hydrogen bond distances $(\AA)$ and angles $\left({ }^{\circ}\right)$ for $\mathbf{2} . \quad$ S21 
(a)

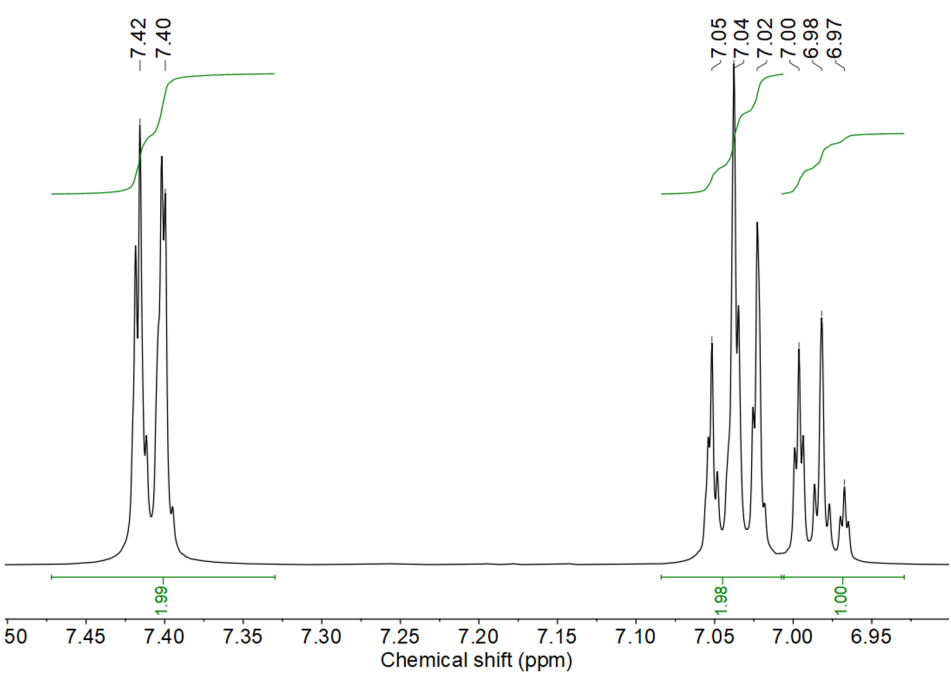

(b)

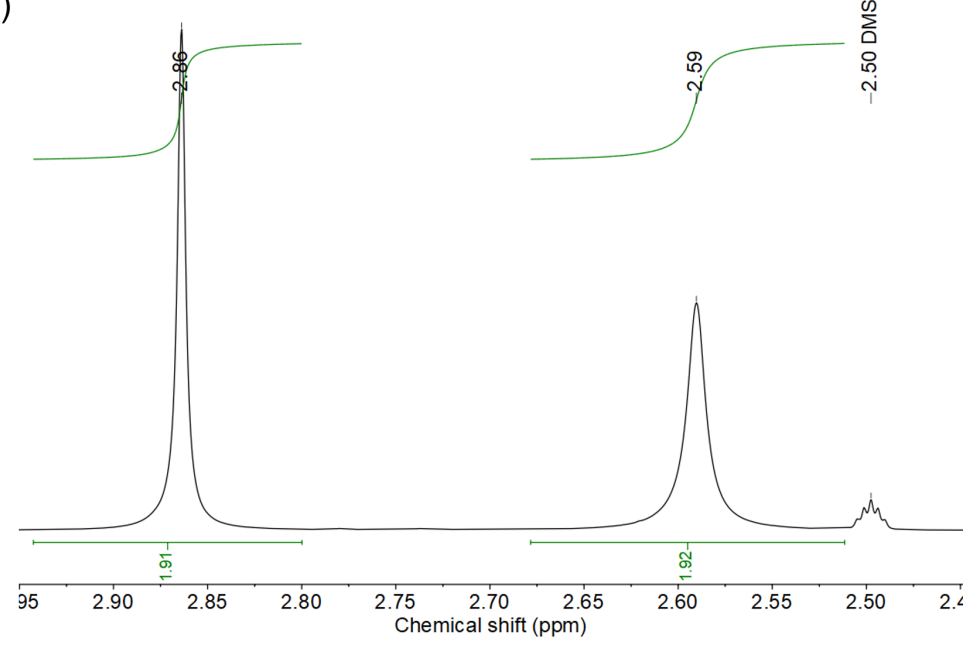

Figure $\mathrm{S} 1$ Integration of the ${ }^{1} \mathrm{H}$ NMR resonances of 1 in DMSO- $d_{6}$ is consistent with the structural formula $\left(\mathrm{C}_{2} \mathrm{H}_{8} \mathrm{~N}_{2}\right) \mathrm{Pb}\left(\mathrm{SeC}_{6} \mathrm{H}_{5}\right)_{2}$. (a) Resonances from protons of the phenyl groups. (b) Resonances from protons of the coordinated ethylenediamine. 


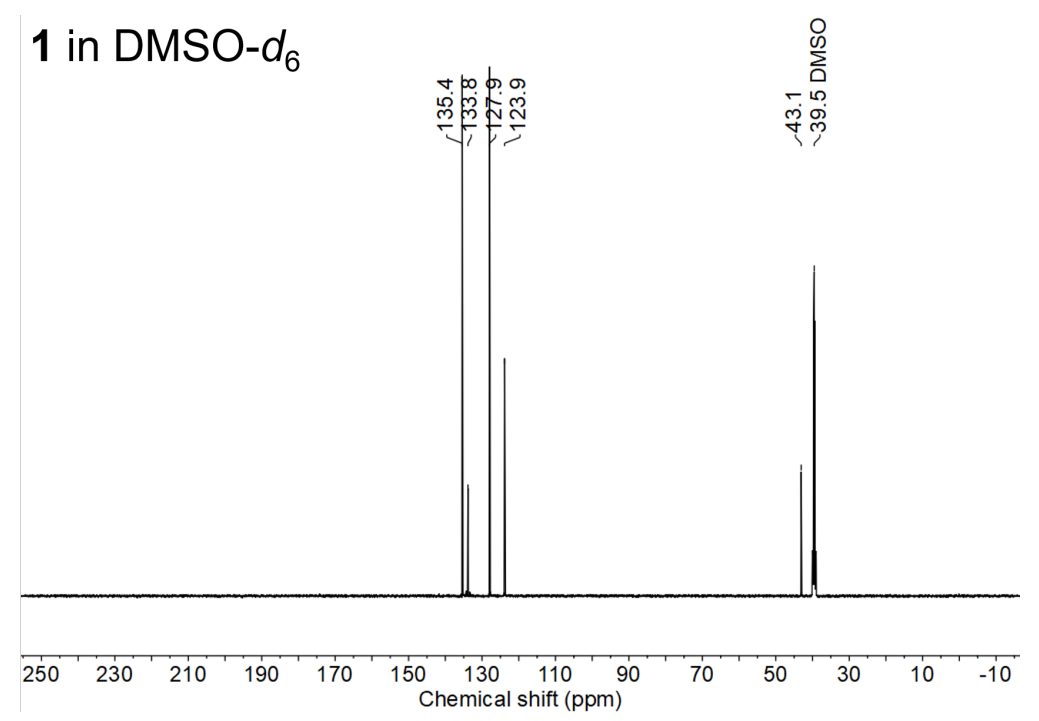

Figure $\mathrm{S} 2{ }^{13} \mathrm{C}$ NMR spectrum of 1 in DMSO- $d_{6}$ is consistent with the structure of ethylenediamine coordinated lead(II) phenylselenolate.

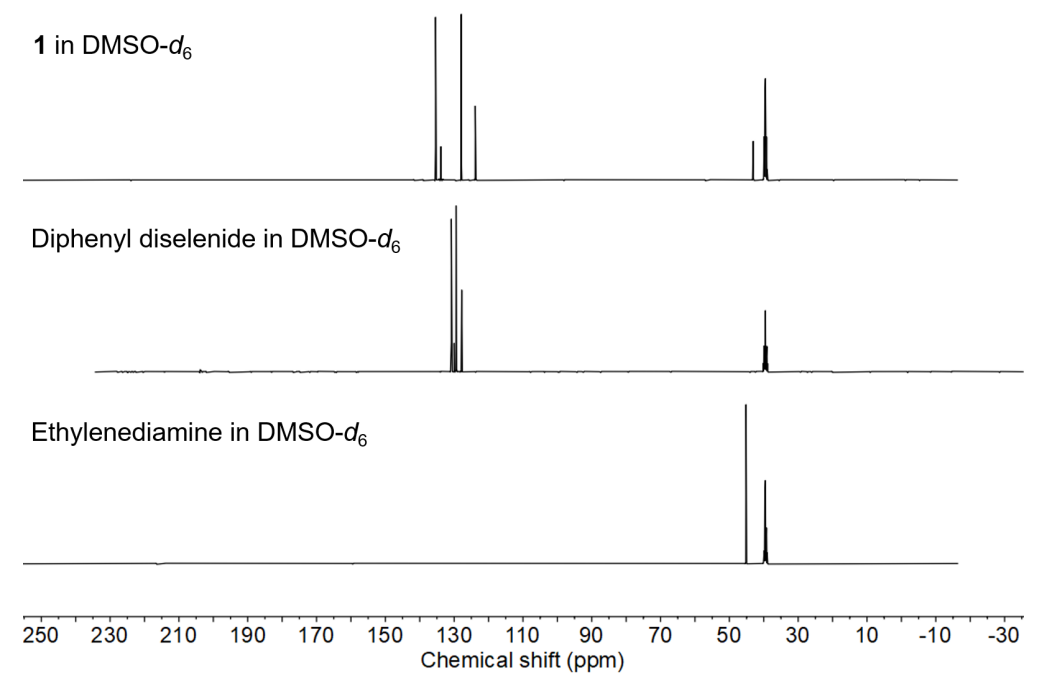

Figure S3 Stacked ${ }^{13}$ C NMR spectra of 1, diphenyl diselenide, and ethylenediamine in DMSO- $d_{6}$. 


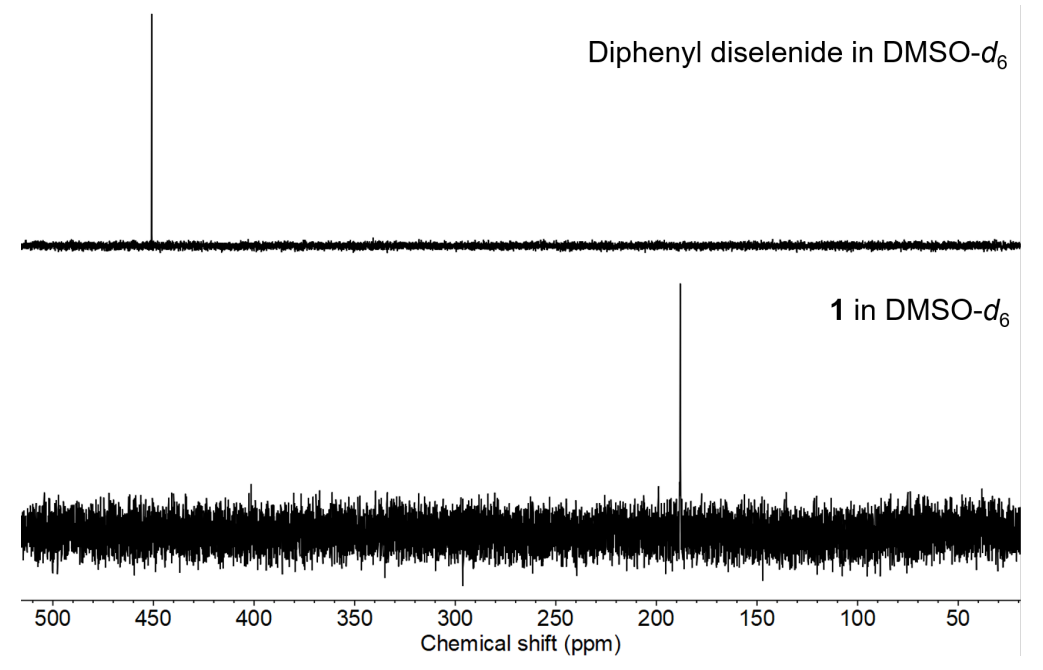

Figure $54{ }^{77}$ Se NMR spectrum of 1 and diphenyl diselenide in DMSO- $d_{6}$. The selenium nuclei are observed to exist in a single chemical environment for 1 consistent with the structure of ethylenediamine coordinated lead(II) phenylselenolate. Moreover, the ${ }^{77}$ Se resonance for 1 appears upfield as compared to diphenyl diselenide.

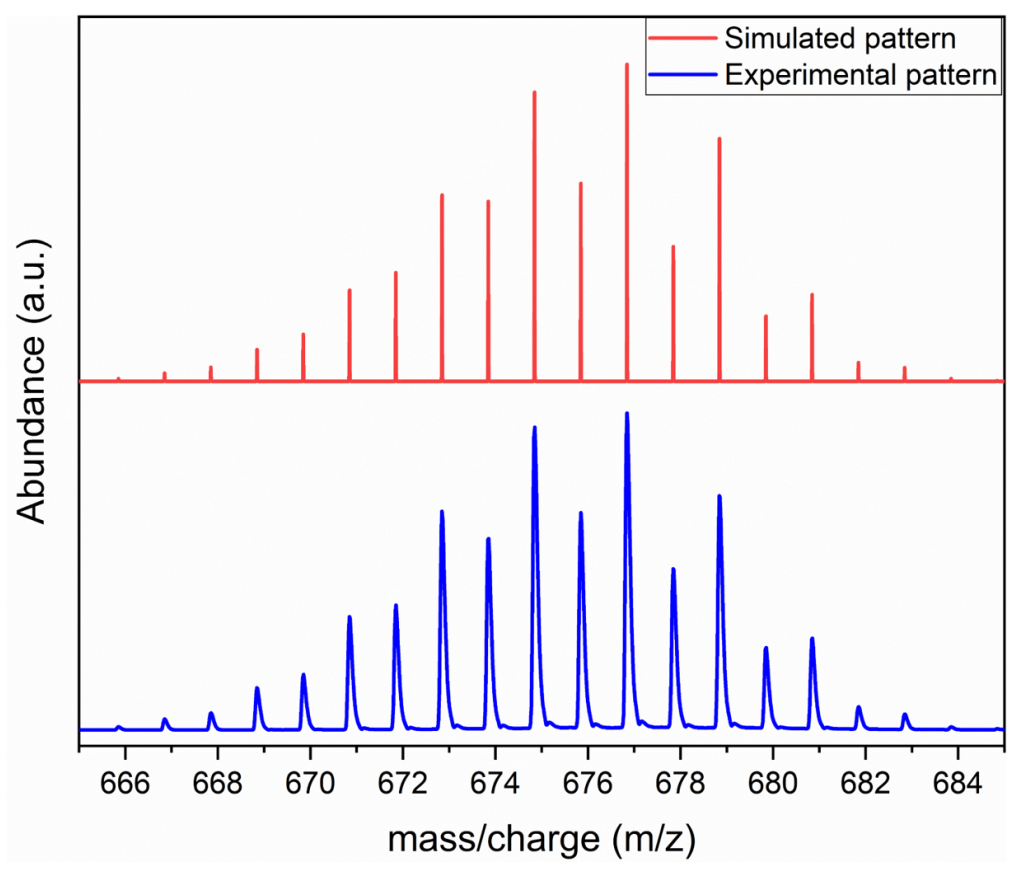

Figure S5 Comparison of the experimental and simulated isotopic pattern of the $\left[\mathrm{PbSe}_{3} \mathrm{C}_{18} \mathrm{H}_{15}\right]^{-1}$ ion. 

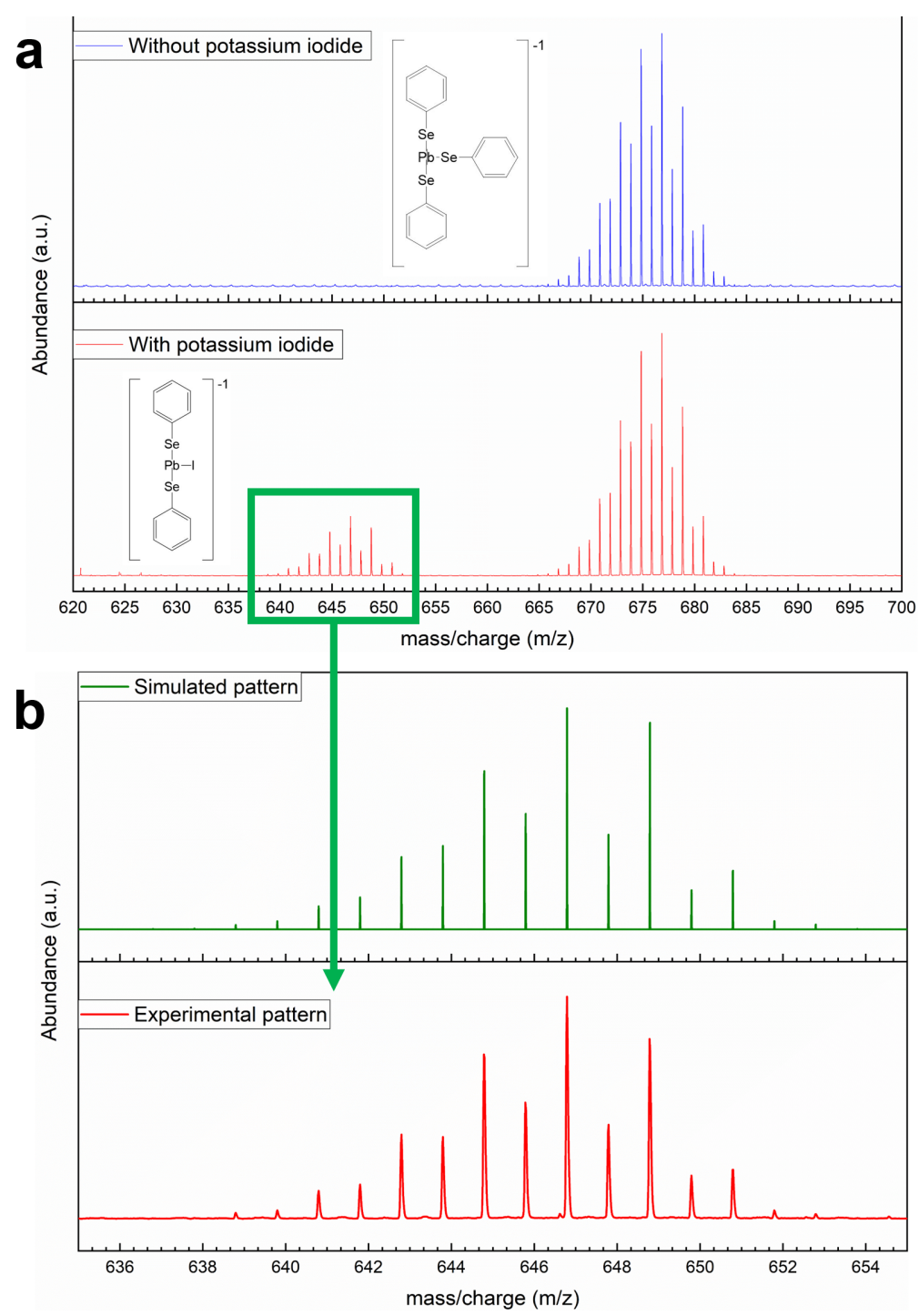

Figure S6 Comparison of mass spectra of the PbSe precursor in ethylenediamine (a) with and without the addition of potassium iodide. (b) Simulated and experimental pattern of ion $\left[\mathrm{PbSe}_{2} \mathrm{C}_{12} \mathrm{H}_{10} \mathrm{I}^{-1}\right.$. 


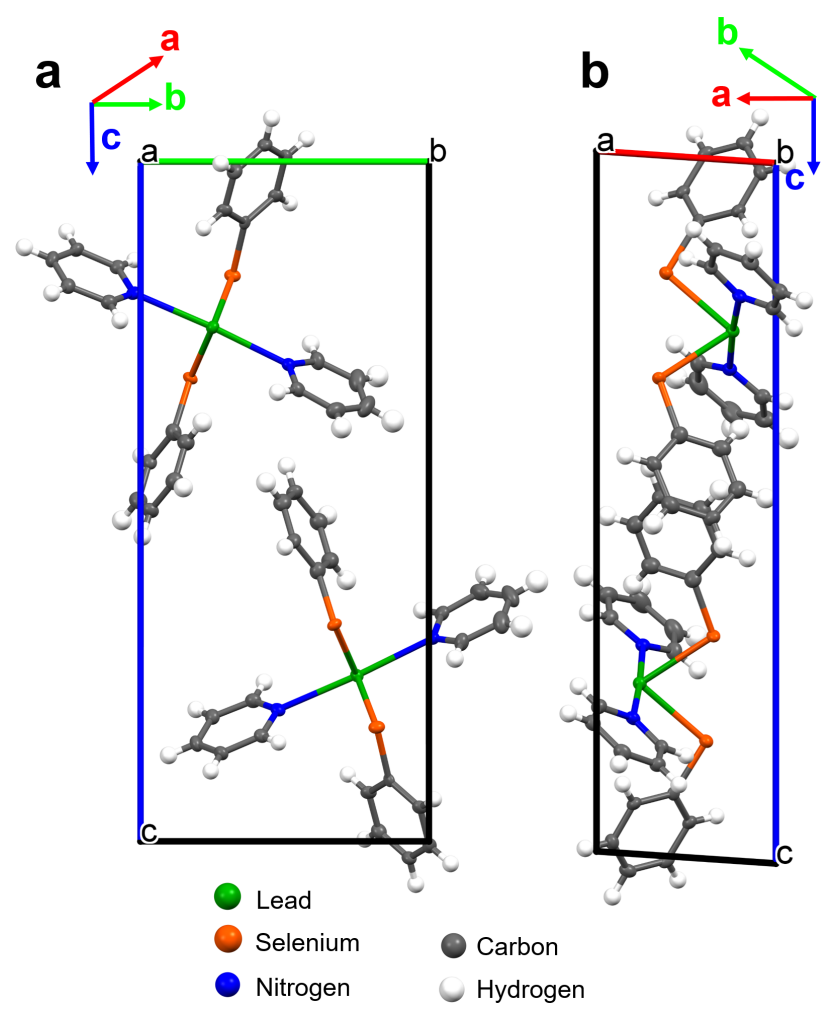

Figure S7 Packing in the crystal structure of 2. The unit cell is comprised of two formula units as seen in (a) and the two units counterbalance each other in orientation as seen in (b). For clarity, crystal disorder is not shown here, but can be viewed in Figure 3 of the main text. (a) Unit cell viewed along the ' $a$ ' direction (b) Unit cell viewed along the ' $b$ ' direction. 
(a) Aged 2 in DMSO-d

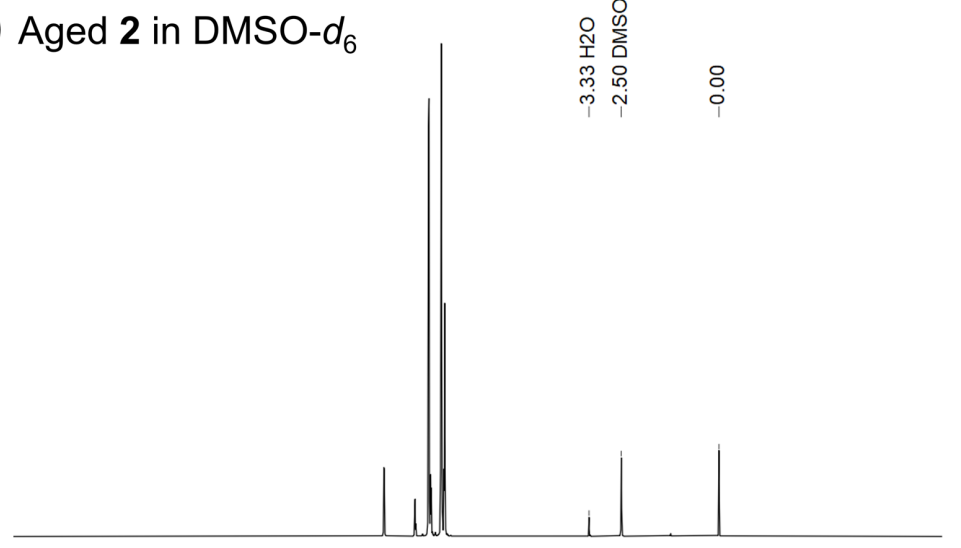

\begin{tabular}{llllllllllllllllllllllllll}
\hline 8 & 17 & 16 & 15 & 14 & 13 & 12 & 11 & 10 & 9 & $\begin{array}{c}8 \\
8\end{array}$ & 7 & 6 & 5 & 4 & 3 & 2 & 1 & 0 & -1 & -2 & -3 & -4 & -5 \\
Chemical shift (ppm)
\end{tabular}

(b)

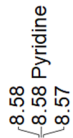

를

$\infty \infty \infty$

inj

$\sqrt{ }$

$\sim$

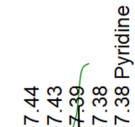

مَ
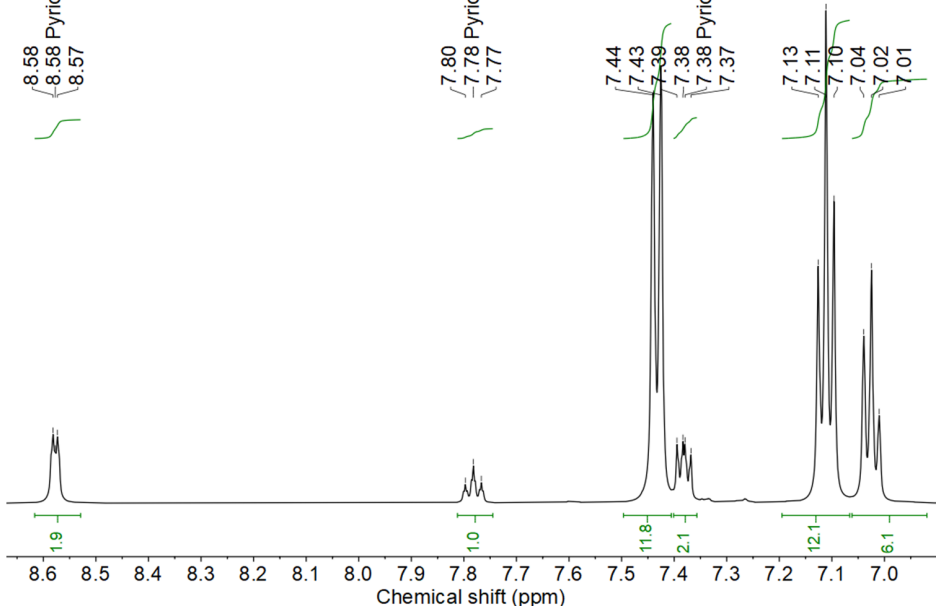

Figure $S 8{ }^{1} \mathrm{H}$ NMR spectrum of aged 2 dissolved in DMSO- $d_{6}$ shows the presence of free pyridine which is likely due to pyridine ligand substitution by dimethyl sulfoxide on lead(II) phenylselenolate complex. (a) Complete ${ }^{1} \mathrm{H}$ NMR spectrum (b) Magnified image of ${ }^{1} \mathrm{H}$ NMR spectrum from 6.9 to $8.7 \mathrm{ppm}$. 
(a)
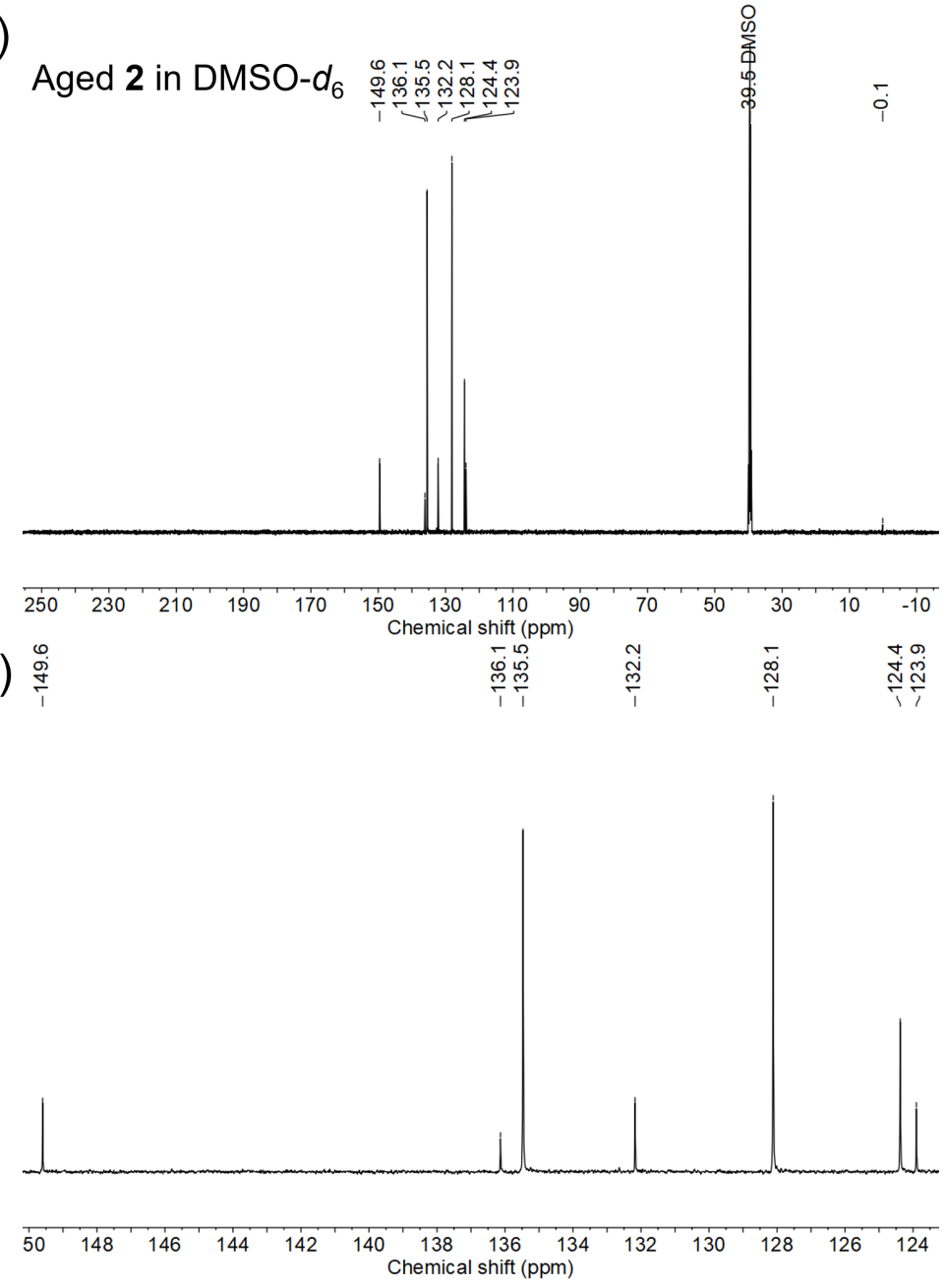

Figure S9 ${ }^{13} \mathrm{C}$ NMR spectrum of aged 2 dissolved in DMSO- $d_{6}$. (a) Complete ${ }^{13} \mathrm{C}$ NMR spectrum. (b) Magnified image of the same ${ }^{13} \mathrm{C}$ NMR spectrum from 123 to $150 \mathrm{ppm}$.

\section{Discussion S1: Crystal structure of 3 showing 1-D molecular chains}

Single-crystal XRD analysis was performed on single crystal specimens of $\mathbf{3}$, which were obtained by growing crystals from the PbSe precursor prepared in DMSO. This analysis revealed the presence of 1-D chains of $\mathrm{Pb}(\mathrm{SePh})_{2}$ in the central region of the unit cell of 3 . The schematic of the 1- $\mathrm{D}$ chain (Figure S8) shows that the $\mathrm{Pb}(\mathrm{SePh})_{2}$ units are $\mu_{2}$-bridged by one of the phenylselenolate ligands ( $\mathrm{Se} 2$ - phenyl group) whereas the other phenylselenolate ligand (Se1 phenyl group) acts as a terminal ligand. The origin of the layered structure can be attributed to the secondary $\mathrm{Pb}$-Se bonds between the parallel chains. The edge region of the unit cell exhibited both inversion twinning and disorder, which hindered the solution of the complete crystal structure. 


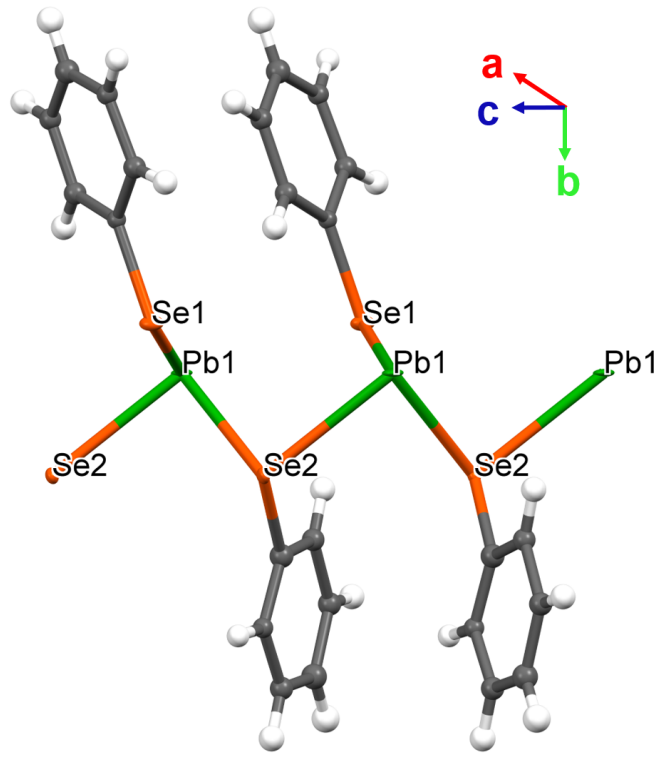

Figure S10 Schematic of the 1-D chains in the central region of the unit cell for the crystal structure of 3 , which is composed of $\mathrm{Pb}(\mathrm{SePh})_{2}$ units.

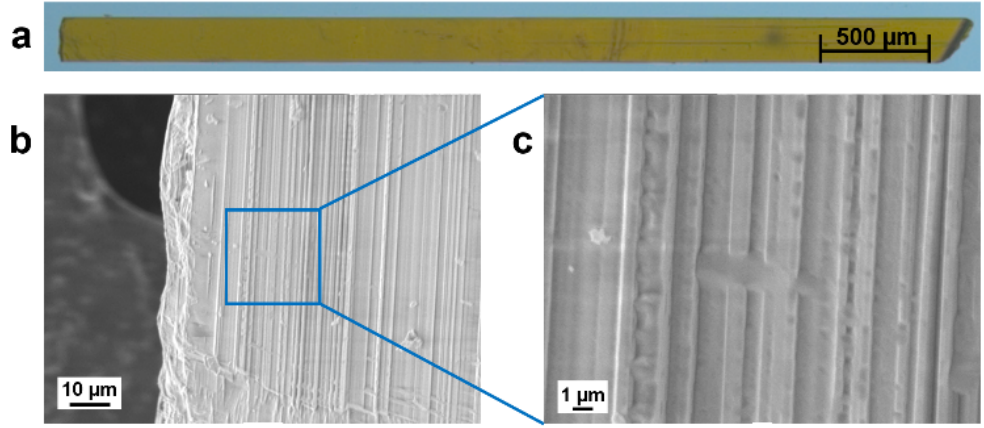

Figure S11 a) Optical micrograph of a representative specimen of a single crystal of $\mathbf{3}$ showing elongation along one of its directions. The crystal's color is orange-yellow in thicker specimens and yellow in thinner specimens. b) and c) SEM images showing the stacking of layers in the single crystal of 3. The layered structures in part (a) exist in the plane of the image (i.e. the layer stacking direction is perpendicular to the page). 

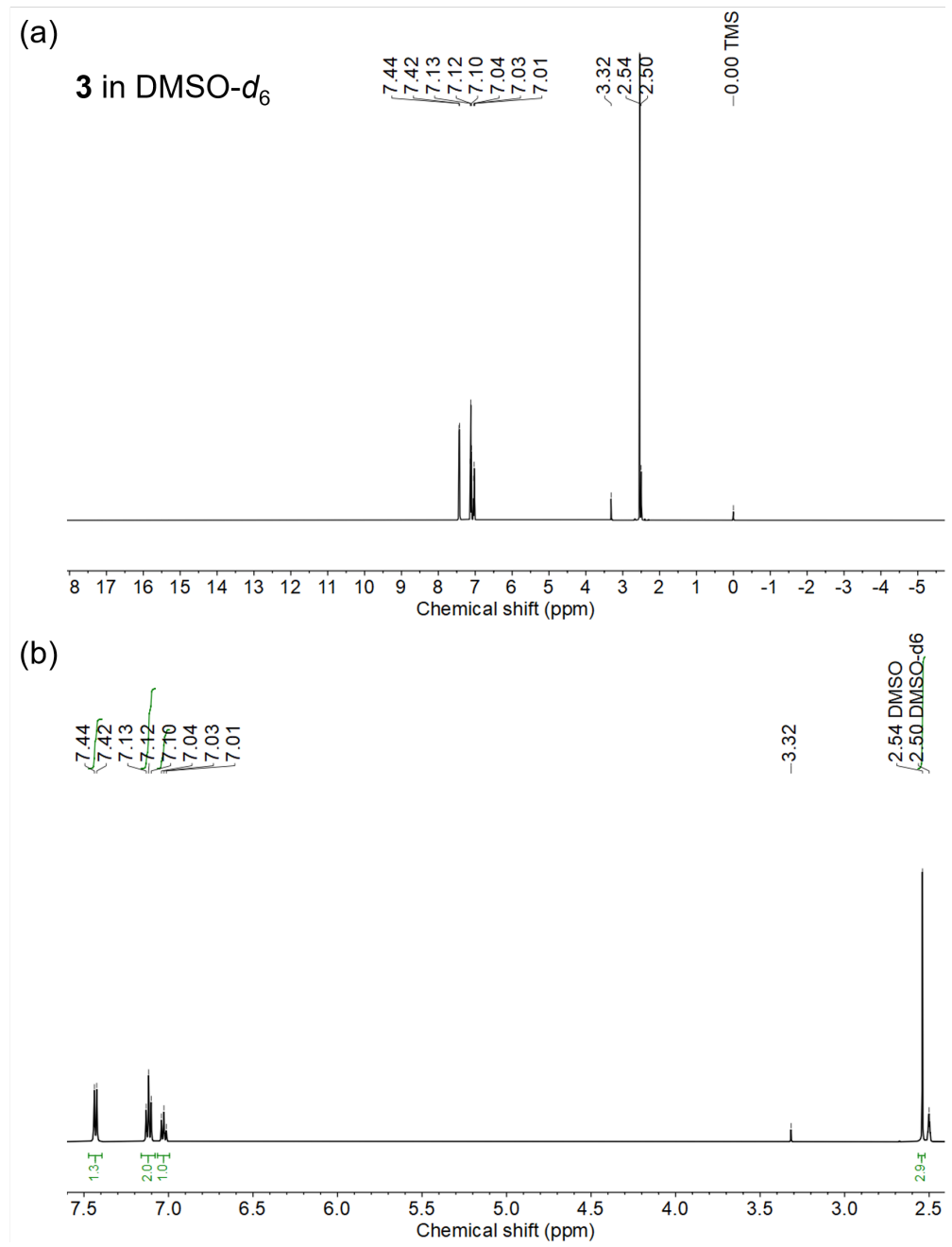

Figure S12 ${ }^{1} \mathrm{H}$ NMR spectrum of 3 dissolved in DMSO- $d_{6}$. (a) Complete ${ }^{1} \mathrm{H}$ NMR spectrum. (b) Magnified image of ${ }^{1} \mathrm{H}$ NMR spectrum from 2.4 to $7.6 \mathrm{ppm}$.

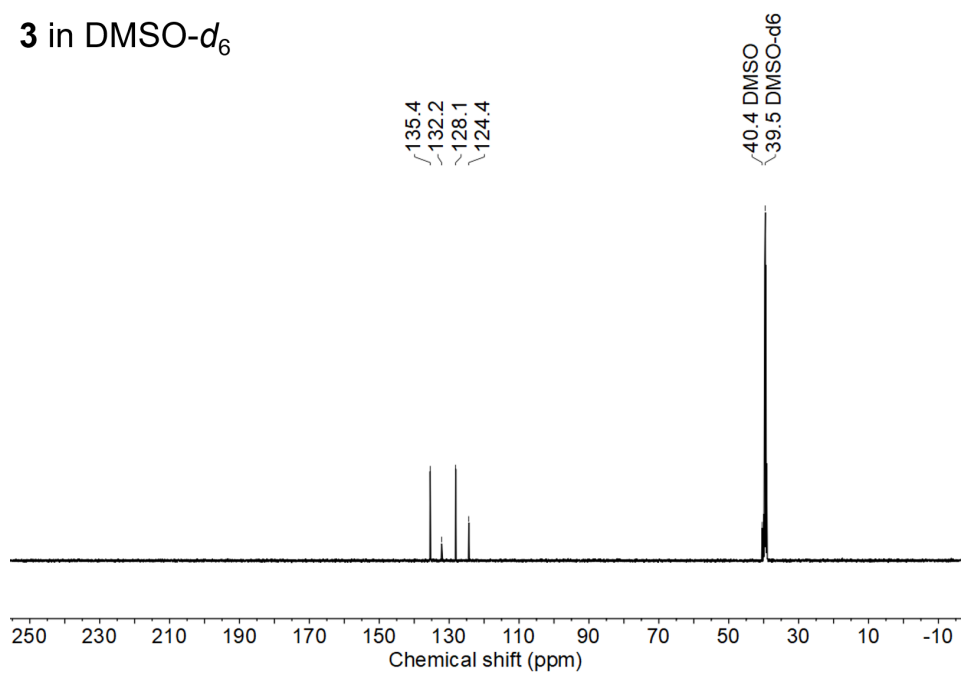

Figure $\mathrm{S} 13{ }^{13} \mathrm{C}$ NMR spectrum of 3 dissolved in DMSO- $d_{6}$. 
Table S1 Additional details of data collection and refinement for $\mathbf{2}$.

\begin{tabular}{|l|l|}
\hline Theta range for data collection & 1.92 to $25.36^{\circ}(0.83 \AA$ resolution $)$ \\
\hline Index ranges & $-6<=\mathrm{h}<=6,-10<=\mathrm{k}<=10,-25<=\mathrm{l}<=25$ \\
\hline $\begin{array}{l}\text { Coverage of independent } \\
\text { reflections }\end{array}$ & $100 \%$ \\
\hline Absolute structure parameter & $-0.0(0)$ \\
\hline Data / restraints / parameters & $3871 / 181 / 272$ \\
\hline Largest diff. peak and hole & 1.479 and $-0.582 \mathrm{e} \AA^{-3}$ \\
\hline R.M.S. deviation from mean & $0.112 \mathrm{e} \AA^{-3}$ \\
\hline Max. and min. transmission & 0.3510 and 0.1920 \\
\hline Weighting scheme & $\begin{array}{l}\mathrm{w}=1 /\left[\sigma^{2}\left(\mathrm{~F}_{\mathrm{o}}{ }^{2}\right)+(0.0315 \mathrm{P})^{2}+1.7019 \mathrm{P}\right] \\
\text { where } \mathrm{P}=\left(\mathrm{F}_{\mathrm{o}}{ }^{2}+2 \mathrm{~F}_{\mathrm{c}}{ }^{2}\right) / 3\end{array}$ \\
\hline
\end{tabular}

Table S2 Atomic coordinates and equivalent isotropic atomic displacement parameters $\left(\AA^{2}\right)$ for 2. $U(e q)$ is defined as one-third of the trace of the orthogonalized $U_{i j}$ tensor.

$$
x / a \quad y / b \quad z / c \quad U(e q)
$$

Pb1 $0.24106(5) \quad 0.25006(4) \quad 0.24377(2) 0.01884(10)$

Se1 $0.61137(16) 0.31883(12) 0.16783(4) \quad 0.0221(2)$

C1 $0.4295(15) \quad 0.3619(9) \quad 0.0899(4) \quad 0.0168(18)$

C2 $0.5188(16) \quad 0.4683(10) \quad 0.0501(4) \quad 0.0214(19)$

C3 $0.3908(17) \quad 0.5022(10) \quad 0.9934(4) \quad 0.024(2)$

C4 $0.1741(18) \quad 0.4313(11) \quad 0.9754(4) \quad 0.025(2)$

C5 $0.0855(16) \quad 0.3265(11) \quad 0.0148(4) \quad 0.0239(19)$

C6 $0.2095(16) \quad 0.2907(10) \quad 0.0717(4) \quad 0.023(2)$

Se2 $0.6515(19) \quad 0.1725(10) \quad 0.3187(3) \quad 0.0192(8)$ 


\begin{tabular}{|c|c|c|c|c|}
\hline & $x / a$ & $y / b$ & $z / c$ & $U(e q)$ \\
\hline C7 & $0.5376(13)$ & $0.1142(7)$ & $0.3981(3)$ & $0.030(4)$ \\
\hline $\mathrm{C} 8$ & $0.3034(14)$ & $0.1571(11)$ & $0.4131(4)$ & $0.033(5)$ \\
\hline C9 & $0.2161(17)$ & $0.1175(13)$ & $0.4701(4)$ & $0.033(5)$ \\
\hline C10 & $0.357(2)$ & $0.0340(12)$ & $0.5132(4)$ & $0.028(4)$ \\
\hline C11 & $0.588(2)$ & $0.9920(13)$ & $0.4984(4)$ & $0.034(5)$ \\
\hline 12 & $0.6807(15)$ & $0.0307(12)$ & $0.4418(4)$ & $0.025(4)$ \\
\hline $2^{\prime}$ & $0.620(2)$ & $0.1619(13)$ & $0.3280(4)$ & $0.0192(8)$ \\
\hline$C 7^{\prime}$ & $0.5050(13)$ & $0.2572(9)$ & $0.4008(3)$ & $0.016(3)$ \\
\hline C8' & $0.2632(14)$ & $0.2344(13)$ & $0.4156(4)$ & $0.021(4)$ \\
\hline C9' & $0.1744(15)$ & $0.3019(15)$ & $0.4676(5)$ & $0.024(5)$ \\
\hline $10^{\prime}$ & $0.323(2)$ & $0.3924(12)$ & $0.5062(4)$ & $0.025(5)$ \\
\hline $11^{\prime}$ & $0.5608(19)$ & $0.4152(13)$ & $0.4915(5)$ & $0.028(5)$ \\
\hline C12' & $0.6542(14)$ & $0.3495(12)$ & $0.4395(5)$ & $0.017(4)$ \\
\hline N1 & $0.2027(14)$ & $0.9762(9)$ & $0.1936(3)$ & $0.0218(16)$ \\
\hline C13 & $0.3629(17)$ & $0.9192(10)$ & $0.1552(4)$ & $0.0217(19)$ \\
\hline C14 & $0.3406(16)$ & $0.7794(10)$ & $0.1297(4)$ & $0.023(2)$ \\
\hline C15 & $0.1442(18)$ & $0.6934(11)$ & $0.1437(4)$ & $0.027(2)$ \\
\hline C16 & $0.9783(15)$ & $0.7490(16)$ & $0.1831(4)$ & $0.0250(17)$ \\
\hline C17 & $0.0139(18)$ & $0.8904(11)$ & $0.2069(4)$ & $0.025(2)$ \\
\hline N2 & $0.2581(13)$ & $0.5132(8)$ & $0.2987(3)$ & $0.0200(16)$ \\
\hline C18 & $0.0760(18)$ & $0.5455(11)$ & $0.3352(4)$ & $0.025(2)$ \\
\hline C19 & $0.063(2)$ & $0.6772(14)$ & $0.3664(5)$ & $0.037(2)$ \\
\hline C20 & $0.244(2)$ & $0.7827(12)$ & $0.3604(6)$ & $0.045(3)$ \\
\hline & (18) & $0.7511(19)$ & 0.322 & $0.042(2)$ \\
\hline
\end{tabular}




$\begin{array}{ccccc} & \mathbf{x} / \mathbf{a} & \mathbf{y} / \mathbf{b} & z / c & U(e q) \\ \mathrm{C22} & 0.4324(18) & 0.6136(11) & 0.2933(5) & 0.028(2)\end{array}$

Table S3 Bond lengths ( $\AA$ ) for $\mathbf{2}$.

\begin{tabular}{|c|c|c|c|}
\hline $\mathrm{Pb} 1-\mathrm{N} 2$ & $2.654(8)$ & $\mathrm{Pb} 1-\mathrm{N} 1$ & $2.702(8)$ \\
\hline Pb1-Se1 & $2.7540(9)$ & $\mathrm{Pb} 1-\mathrm{Se} 2$ & $2.762(11)$ \\
\hline Pb1-Se2' & $2.770(13)$ & Se1-C1 & $1.921(9)$ \\
\hline C1-C2 & $1.395(13)$ & C1-C6 & $1.399(12)$ \\
\hline $\mathrm{C} 2-\mathrm{C} 3$ & $1.391(13)$ & $\mathrm{C} 2-\mathrm{H} 2$ & 0.95 \\
\hline C3-C4 & $1.382(13)$ & $\mathrm{C} 3-\mathrm{H} 3$ & 0.95 \\
\hline C4-C5 & $1.379(14)$ & $\mathrm{C} 4-\mathrm{H} 4$ & 0.95 \\
\hline C5-C6 & $1.390(13)$ & $\mathrm{C} 5-\mathrm{H} 5$ & 0.95 \\
\hline $\mathrm{C} 6-\mathrm{H} 6$ & 0.95 & Se2-C7 & 1.9207 \\
\hline C7-C8 & 1.3989 & $\mathrm{C} 7-\mathrm{C} 12$ & 1.3997 \\
\hline C8-C9 & 1.3849 & $\mathrm{C} 8-\mathrm{H} 8$ & 0.95 \\
\hline C9-C10 & 1.3862 & $\mathrm{C} 9 \mathrm{-H} 9$ & 0.95 \\
\hline C10-C11 & 1.3794 & $\mathrm{C} 10-\mathrm{H} 10$ & 0.95 \\
\hline C11-C12 & 1.3877 & C11-H11 & 0.95 \\
\hline $\mathrm{C} 12-\mathrm{H} 12$ & 0.95 & Se2'-C7' & 1.9207 \\
\hline C7'-C8' & 1.3989 & $C 7^{\prime}-\mathrm{C} 12^{\prime}$ & 1.3997 \\
\hline C8'-C9' & 1.3849 & $\mathrm{C} 8^{\prime}-\mathrm{H} 8^{\prime}$ & 0.95 \\
\hline C9'-C10' & 1.3861 & C9'-H9' & 0.95 \\
\hline C10'-C11' & 1.3794 & C10'-H10' & 0.95 \\
\hline C11'-C12' & 1.3877 & C11'-H11' & 0.95 \\
\hline
\end{tabular}




$\begin{array}{cccc}\mathrm{C} 12^{\prime}-\mathrm{H} 12^{\prime} & 0.95 & \mathrm{~N} 1-\mathrm{C} 17 & 1.339(12) \\ \mathrm{N} 1-\mathrm{C} 13 & 1.345(12) & \mathrm{C} 13-\mathrm{C} 14 & 1.379(13) \\ \mathrm{C} 13-\mathrm{H} 13 & 0.95 & \mathrm{C} 14-\mathrm{C} 15 & 1.378(13) \\ \mathrm{C} 14-\mathrm{H} 14 & 0.95 & \mathrm{C} 15-\mathrm{C} 16 & 1.375(14) \\ \mathrm{C} 15-\mathrm{H} 15 & 0.95 & \mathrm{C} 16-\mathrm{C} 17 & 1.386(16) \\ \mathrm{C} 16-\mathrm{H} 16 & 0.95 & \mathrm{C} 17-\mathrm{H} 17 & 0.95 \\ \mathrm{~N} 2-\mathrm{C} 22 & 1.329(12) & \mathrm{N} 2-\mathrm{C} 18 & 1.339(12) \\ \mathrm{C} 18-\mathrm{C} 19 & 1.370(15) & \mathrm{C} 18-\mathrm{H} 18 & 0.95 \\ \mathrm{C} 19-\mathrm{C} 20 & 1.389(17) & \mathrm{C} 19-\mathrm{H} 19 & 0.95 \\ \mathrm{C} 20-\mathrm{C} 21 & 1.388(16) & \mathrm{C} 20-\mathrm{H} 20 & 0.95 \\ \mathrm{C} 21-\mathrm{C} 22 & 1.388(18) & \mathrm{C} 21-\mathrm{H} 21 & 0.95 \\ \mathrm{C} 22-\mathrm{H} 22 & 0.95 & & \end{array}$

Table S4 Bond angles $\left({ }^{\circ}\right)$ for 2.

$\begin{array}{cccc}\text { N2-Pb1-N1 } & 176.1(2) & \text { N2-Pb1-Se1 } & 93.01(16) \\ \text { N1-Pb1-Se1 } & 90.84(17) & \text { N2-Pb1-Se2 } & 88.2(2) \\ \text { N1-Pb1-Se2 } & 92.0(2) & \text { Se1-Pb1-Se2 } & 78.14(14) \\ \text { N2-Pb1-Se2' } & 88.2(3) & \text { N1-Pb1-Se2' } & 91.6(3) \\ \text { Se1-Pb1-Se2' } & 84.12(18) & \text { C1-Se1-Pb1 } & 101.3(2) \\ \text { C2-C1-C6 } & 118.7(8) & \text { C2-C1-Se1 } & 119.0(6) \\ \text { C6-C1-Se1 } & 122.3(7) & \text { C3-C2-C1 } & 120.3(8) \\ \text { C3-C2-H2 } & 119.8 & \text { C1-C2-H2 } & 119.8 \\ \text { C4-C3-C2 } & 120.9(9) & \text { C4-C3-H3 } & 119.5 \\ \text { C2-C3-H3 } & 119.5 & \text { C5-C4-C3 } & 118.7(9)\end{array}$




\begin{tabular}{|c|c|c|c|}
\hline C5-C4-H4 & 120.7 & C3-C4-H4 & 120.7 \\
\hline C4-C5-C6 & $121.5(9)$ & $\mathrm{C} 4-\mathrm{C} 5-\mathrm{H} 5$ & 119.2 \\
\hline C6-C5-H5 & 119.2 & C5-C6-C1 & $119.8(8)$ \\
\hline C5-C6-H6 & 120.1 & C1-C6-H6 & 120.1 \\
\hline C7-Se2-Pb1 & $106.3(3)$ & C8-C7-C12 & 118.6 \\
\hline C8-C7-Se2 & 118.9 & C12-C7-Se2 & 122.4 \\
\hline $\mathrm{C} 9-\mathrm{C} 8-\mathrm{C} 7$ & 120.4 & $\mathrm{C} 9-\mathrm{C} 8-\mathrm{H} 8$ & 119.8 \\
\hline C7-C8-H8 & 119.8 & C8-C9-C10 & 121.0 \\
\hline С8-С9-H9 & 119.5 & C10-C9-H9 & 119.5 \\
\hline C11-C10-C9 & 118.6 & C11-C10-H10 & 120.7 \\
\hline C9-C10-H10 & 120.7 & $\mathrm{C} 10-\mathrm{C} 11-\mathrm{C} 12$ & 121.6 \\
\hline C10-C11-H11 & 119.2 & C12-C11-H11 & 119.2 \\
\hline C11-C12-C7 & 119.8 & $\mathrm{C} 11-\mathrm{C} 12-\mathrm{H} 12$ & 120.1 \\
\hline C7-C12-H12 & 120.1 & C7'-Se2'-Pb1 & $97.1(3)$ \\
\hline$C 8^{\prime}-C 7^{\prime}-C 12^{\prime}$ & 118.6 & C8'-C7'-Se2' & 118.9 \\
\hline C12'-C7'-Se2' & 122.4 & $\mathrm{C} 9^{\prime}-\mathrm{C} 8^{\prime}-\mathrm{C} 7^{\prime}$ & 120.4 \\
\hline $\mathrm{C} 9^{\prime}-\mathrm{C} 8^{\prime}-\mathrm{H} 8^{\prime}$ & 119.8 & $\mathrm{C} 7^{\prime}-\mathrm{C} 8^{\prime}-\mathrm{H} 8^{\prime}$ & 119.8 \\
\hline C8'-C9'-C10' & 121.0 & C8'-C9'-H9' & 119.5 \\
\hline C10'-C9'-H9' & 119.5 & C11'-C10'-C9' & 118.6 \\
\hline C11'-C10'-H10' & 120.7 & C9'-C10'-H10' & 120.7 \\
\hline C10'-C11'-C12' & 121.6 & C10'-C11'-H11' & 119.2 \\
\hline C12'-C11'-H11' & 119.2 & $\mathrm{C} 11^{\prime}-\mathrm{C} 12^{\prime}-\mathrm{C} 7^{\prime}$ & 119.8 \\
\hline C11'-C12'-H12' & 120.1 & $\mathrm{C} 7^{\prime}-\mathrm{C} 12^{\prime}-\mathrm{H} 12^{\prime}$ & 120.1 \\
\hline C17-N1-C13 & $116.9(8)$ & C17-N1-Pb1 & $119.2(6)$ \\
\hline C13-N1-Pb1 & $123.9(6)$ & N1-C13-C14 & $123.3(9)$ \\
\hline
\end{tabular}




\begin{tabular}{|c|c|c|c|}
\hline $\mathrm{N} 1-\mathrm{C} 13-\mathrm{H} 13$ & 118.4 & C14-C13-H13 & 118.4 \\
\hline C15-C14-C13 & $118.8(9)$ & C15-C14-H14 & 120.6 \\
\hline C13-C14-H14 & 120.6 & C16-C15-C14 & $119.0(10)$ \\
\hline C16-C15-H15 & 120.5 & C14-C15-H15 & 120.5 \\
\hline C15-C16-C17 & $118.7(10)$ & C15-C16-H16 & 120.7 \\
\hline C17-C16-H16 & 120.7 & N1-C17-C16 & 123.3(9) \\
\hline N1-C17-H17 & 118.3 & C16-C17-H17 & 118.3 \\
\hline C22-N2-C18 & $118.0(8)$ & $\mathrm{C} 22-\mathrm{N} 2-\mathrm{Pb} 1$ & $125.4(6)$ \\
\hline C18-N2-Pb1 & 116.6(6) & N2-C18-C19 & $122.5(10)$ \\
\hline N2-C18-H18 & 118.8 & C19-C18-H18 & 118.8 \\
\hline C18-C19-C20 & 119.5(10) & C18-C19-H19 & 120.2 \\
\hline C20-C19-H19 & 120.2 & C21-C20-C19 & $118.4(11)$ \\
\hline C21-C2O-H2O & 120.8 & C19-C2O-H2O & 120.8 \\
\hline C22-C21-C20 & 117.9(12) & $\mathrm{C} 22-\mathrm{C} 21-\mathrm{H} 21$ & 121.0 \\
\hline C2O-C21-H21 & 121.0 & N2-C22-C21 & $123.5(10)$ \\
\hline $\mathrm{N} 2-\mathrm{C} 22-\mathrm{H} 22$ & 118.2 & $\mathrm{C} 21-\mathrm{C} 22-\mathrm{H} 22$ & 118.2 \\
\hline
\end{tabular}

Table S5 Torsion angles $\left({ }^{\circ}\right)$ for $\mathbf{2}$.

$\begin{array}{lccc}\text { C6-C1-C2-C3 } & -0.1(13) & \text { Se1-C1-C2-C3 } & -179.8(7) \\ \text { C1-C2-C3-C4 } & -0.2(14) & \text { C2-C3-C4-C5 } & 0.4(14) \\ \text { C3-C4-C5-C6 } & -0.3(14) & \text { C4-C5-C6-C1 } & 0.0(14) \\ \text { C2-C1-C6-C5 } & 0.3(13) & \text { Se1-C1-C6-C5 } & 179.9(7) \\ \text { C12-C7-C8-C9 } & 0 & \text { Se2-C7-C8-C9 } & -179.7 \\ \text { C7-C8-C9-C10 } & -0.4 & \text { C8-C9-C10-C11 } & 0.6\end{array}$




$\begin{array}{ccccc}\text { C9-C10-C11-C12 } & -0.3 & \text { C10-C11-C12-C7 } & -0.1 \\ \text { C8-C7-C12-C11 } & 0.3 & \text { Se2-C7-C12-C11 } & -180.0 \\ \text { C12'-C7'-C8'-C9' } & 0 & \text { Se2'-C7'-C8'-C9' } & -179.7 \\ \text { C7'-C8'-C9'-C10' } & -0.4 & C^{\prime} 8^{\prime}-C 9^{\prime}-C 10^{\prime}-C 11^{\prime} & 0.6 \\ \text { C9'-C10'-C11'-C12' } & -0.3 & \text { C10'-C11'-C12'-C7' } & -0.1 \\ \text { C8'-C7'-C12'-C11' } & 0.3 & \text { Se2'-C7'-C12'-C11' } & -180.0 \\ \text { C17-N1-C13-C14 } & -0.1(13) & \text { Pb1-N1-C13-C14 } & -179.7(7) \\ \text { N1-C13-C14-C15 } & 0.5(14) & \text { C13-C14-C15-C16 } & -0.7(13) \\ \text { C14-C15-C16-C17 } & 0.5(14) & \text { C13-N1-C17-C16 } & 0.0(14) \\ \text { Pb1-N1-C17-C16 } & 179.6(7) & \text { C15-C16-C17-N1 } & -0.2(14) \\ \text { C22-N2-C18-C19 } & 0.3(14) & \text { Pb1-N2-C18-C19 } & 179.8(8) \\ \text { N2-C18-C19-C20 } & 0.2(16) & \text { C18-C19-C20-C21 } & 0.6(17) \\ \text { C19-C20-C21-C22 } & -1.8(16) & \text { C18-N2-C22-C21 } & -1.7(14) \\ \text { Pb1-N2-C22-C21 } & 178.9(8) & \text { C20-C21-C22-N2 } & 2.4(16)\end{array}$

Table S6 Anisotropic atomic displacement parameters $\left(\AA^{2}\right)$ for 2 . The anisotropic atomic displacement factor exponent takes the form: $-2 \pi^{2}\left[h^{2} a^{* 2} U_{11}+\ldots+2 h k a^{*} b^{*} U_{12}\right]$.

$\begin{array}{llllll}U_{11} & U_{22} & U_{33} & U_{23} & U_{13} & U_{12}\end{array}$

Pb1 0.01890(15) 0.01886(15) $0.01870(16)-0.0017(2) 0.00070(10) \quad \begin{gathered}- \\ 0.00024(16)\end{gathered}$

Se1 $0.0196(4) \quad 0.0288(5) \quad 0.0176(5) \quad 0.0016(4) \quad-0.0007(4) \quad-0.0007(4)$

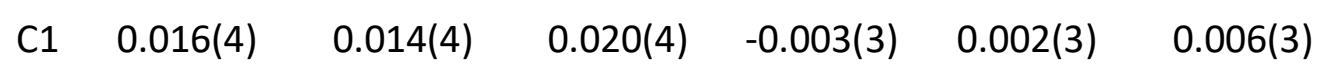

$\begin{array}{lllllll}C 2 & 0.021(4) & 0.025(4) & 0.019(4) & -0.002(3) & 0.006(3) & -0.001(3)\end{array}$

$\begin{array}{lllllll}C 3 & 0.028(4) & 0.021(4) & 0.024(4) & 0.001(3) & 0.009(4) & -0.001(4)\end{array}$

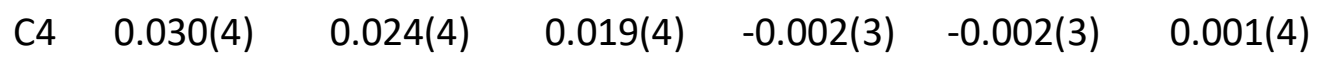




$\begin{array}{ccccccc} & U_{11} & U_{22} & U_{33} & U_{23} & U_{13} & U_{12} \\ \text { C5 } & 0.020(4) & 0.026(4) & 0.026(4) & -0.003(4) & -0.001(3) & 0.000(4) \\ \text { C6 } & 0.022(4) & 0.028(5) & 0.018(4) & 0.000(3) & 0.006(3) & -0.001(3) \\ \text { Se2 } & 0.019(2) & 0.0223(12) & 0.0161(17) & 0.0050(14) & 0.0007(12) & -0.0029(12) \\ \text { C7 } & 0.030(7) & 0.027(7) & 0.032(7) & 0.002(5) & 0.004(5) & -0.002(5) \\ \text { C8 } & 0.038(7) & 0.032(7) & 0.029(7) & 0.001(6) & 0.001(6) & 0.001(6) \\ \text { C9 } & 0.032(7) & 0.034(7) & 0.033(7) & -0.002(6) & 0.005(6) & -0.001(6) \\ \text { C10 } & 0.030(7) & 0.030(7) & 0.024(7) & 0.003(5) & 0.007(5) & 0.001(6) \\ \text { C11 } & 0.034(7) & 0.035(7) & 0.032(7) & 0.002(6) & -0.004(6) & 0.000(6) \\ \text { C12 } & 0.026(6) & 0.024(6) & 0.025(6) & 0.002(5) & -0.004(5) & -0.002(5) \\ \text { Se2' } & 0.019(2) & 0.0223(12) & 0.0161(17) & 0.0050(14) & 0.0007(12) & -0.0029(12) \\ \text { C7' } & 0.017(6) & 0.019(6) & 0.012(5) & 0.003(6) & -0.003(5) & 0.001(6) \\ \text { C8' } & 0.021(6) & 0.023(7) & 0.020(6) & 0.003(6) & 0.001(5) & 0.000(6) \\ \text { C9' } & 0.024(7) & 0.026(7) & 0.023(7) & 0.004(5) & 0.002(5) & 0.001(5) \\ \text { C10' } & 0.030(7) & 0.027(7) & 0.018(7) & 0.000(6) & 0.010(6) & 0.001(6) \\ \text { C11' } & 0.031(7) & 0.028(7) & 0.026(7) & -0.004(6) & -0.003(6) & -0.005(6) \\ \text { C12' } & 0.018(6) & 0.018(7) & 0.016(6) & 0.000(5) & 0.000(5) & -0.005(5) \\ \text { N1 } & 0.022(3) & 0.024(4) & 0.019(3) & -0.003(3) & -0.002(3) & -0.001(3) \\ \text { C13 } & 0.019(4) & 0.024(4) & 0.021(4) & -0.002(3) & 0.000(3) & -0.003(3) \\ \text { C14 } & 0.026(4) & 0.022(5) & 0.021(4) & -0.003(3) & 0.003(3) & 0.004(3) \\ \text { C15 } & 0.032(4) & 0.019(4) & 0.028(4) & -0.004(3) & -0.003(4) & -0.001(4) \\ \text { C16 } & 0.026(4) & 0.023(4) & 0.026(4) & 0.000(5) & -0.001(3) & 0.002(5) \\ \text { C17 } & 0.025(4) & 0.028(4) & 0.023(4) & 0.000(4) & 0.004(3) & 0.001(4) \\ \text { N2 } & 0.022(3) & 0.020(3) & 0.018(3) & 0.001(3) & 0.001(3) & 0.002(3) \\ \text { C18 } & 0.027(4) & 0.023(4) & 0.025(4) & 0.004(3) & 0.003(3) & 0.005(3)\end{array}$




$\begin{array}{ccccccc} & \mathbf{U}_{11} & \mathbf{U}_{22} & \mathbf{U}_{33} & \mathbf{U}_{23} & \mathbf{U}_{13} & \mathbf{U}_{12} \\ \mathrm{C} 19 & 0.041(5) & 0.041(5) & 0.030(5) & -0.004(4) & 0.003(4) & 0.013(4) \\ \mathrm{C} 20 & 0.050(5) & 0.033(6) & 0.050(5) & -0.016(4) & -0.014(4) & 0.012(4) \\ \mathrm{C} 21 & 0.039(4) & 0.030(4) & 0.056(5) & -0.002(6) & -0.012(4) & -0.002(5) \\ \mathrm{C} 22 & 0.026(4) & 0.023(4) & 0.035(5) & 0.003(4) & 0.002(4) & -0.001(4)\end{array}$

Table S7 Hydrogen atomic coordinates and isotropic atomic displacement parameters $\left(\AA^{2}\right)$ for 2.

\begin{tabular}{ccccc} 
& $\mathbf{x} / \mathbf{a}$ & $\mathbf{y} / \mathbf{b}$ & $\mathbf{z} / \mathbf{c}$ & $\mathbf{U}(\mathbf{e q})$ \\
H2 & 0.6677 & 0.5179 & 0.0617 & 0.026 \\
H3 & 0.4533 & 0.5751 & -0.0332 & 0.029 \\
H4 & 0.0879 & 0.4543 & -0.0635 & 0.03 \\
H5 & -0.0637 & 0.2776 & 0.0029 & 0.029 \\
H6 & 0.1450 & 0.2182 & 0.0982 & 0.027 \\
H8 & 0.2037 & 0.2138 & 0.3840 & 0.04 \\
H9 & 0.0572 & 0.1480 & 0.4797 & 0.039 \\
H10 & 0.2960 & 0.0062 & 0.5520 & 0.033 \\
H11 & 0.6866 & -0.0649 & 0.5278 & 0.041 \\
\hline H12 & 0.8406 & 0.0007 & 0.4327 & 0.03 \\
\hline H8' & 0.1593 & 0.1721 & 0.3898 & 0.025 \\
H9' & 0.0095 & 0.2859 & 0.4769 & 0.029 \\
H10' & 0.2614 & 0.4378 & 0.5421 & 0.029 \\
H11' & 0.6635 & 0.4775 & 0.5176 & 0.034 \\
H13 & 0.4981 & -0.0219 & 0.1450 & 0.026
\end{tabular}




$\begin{array}{ccccc} & \mathbf{x} / \mathbf{a} & \mathbf{y} / \mathbf{b} & \mathbf{z} / \mathbf{c} & \mathbf{U}(\mathbf{e q}) \\ \mathrm{H} 14 & 0.4586 & -0.2570 & 0.1030 & 0.028 \\ \mathrm{H} 15 & 0.1237 & -0.4029 & 0.1264 & 0.032 \\ \mathrm{H} 16 & -0.1580 & -0.3083 & 0.1938 & 0.03 \\ \mathrm{H} 17 & -0.1015 & -0.0716 & 0.2340 & 0.03 \\ \mathrm{H} 18 & -0.0491 & 0.4743 & 0.3396 & 0.03 \\ \mathrm{H} 19 & -0.0684 & 0.6963 & 0.3920 & 0.045 \\ \text { H20 } & 0.2394 & 0.8743 & 0.3820 & 0.054 \\ \text { H21 } & 0.5569 & 0.8214 & 0.3158 & 0.051 \\ \text { H22 } & 0.5641 & 0.5899 & 0.2684 & 0.033\end{array}$

Table S8 Hydrogen bond distances $(\AA)$ and angles $\left({ }^{\circ}\right)$ for 2.

Donor-H Acceptor-H Donor-Acceptor Angle

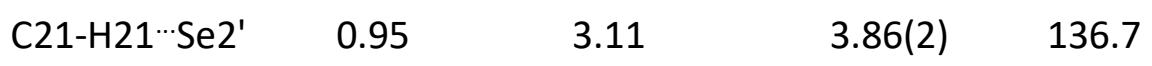

